\title{
Consumo de alcohol, marihuana y cocaína en estudiantes de medicina de la Pontificia Universidad Católica de Chile
}

\author{
Use of alcohol, marijuana and cocaine among medical students of the \\ Pontificia Universidad Católica de Chile
}

María José Calletti G' ${ }^{1}$ Enrique Elsaca M', Valentina Kobus G'1 , Jaime Santander $\mathrm{T}^{2 *}$

\begin{abstract}
Resumen
Introducción: El uso de sustancias en adolescentes y adultos jóvenes es un problema importante en Chile. El objetivo de este trabajo es determinar la prevalencia de consumo de alcohol, marihuana y cocaína entre los estudiantes de medicina de la Pontificia Universidad Católica de Chile (PUC), estudiar su relación con ciertas variables psicosociales y contrastar con estudios previos. Métodos: Se diseñó una encuesta voluntaria y de autoreporte, que incluyó el cuestionario "AUDIT" para consumo de alcohol, preguntas de la encuesta de Servicio Nacional para la Prevención y Rehabilitación del Consumo de Drogas y Alcohol (SENDA) para consumo de marihuana y cocaína, y preguntas sobre características sociodemográficas. Resultados: Respondieron 559 estudiantes de un total de 857 (65,2\%). La prevalencia de consumo de riesgo de alcohol fue $15,1 \%$, mientras que de consumo perjudicial fue $5,2 \%$. La prevalencia de consumo de marihuana fue $57,2 \%$ alguna vez en la vida, 38,2\% el último año, y 18,5\% el último mes. La prevalencia de consumo de cocaína fue 2,8\% alguna vez en la vida, 1,6\% el último año, y $0,5 \%$ el último mes. Se encontraron asociaciones significativas con las variables estudiadas. Conclusiones: Ha aumentado considerablemente el consumo de sustancias entre los estudiantes de medicina de la PUC. No obstante, la prevalencia es menor si se compara con el resto de estudiantes de educación superior chilena y similar a lo reportado en estudios de Norteamérica y Europa.
\end{abstract}

Palabras clave: estudiantes de medicina; alcohol; marihuana; cocaína; trastorno por uso de sustancias; abuso de drogas.

\begin{abstract}
Introduction: The objective of this work is to determine alcohol, cannabis and cocaine use prevalence among medical students from the Pontificia Universidad Católica de Chile, study its relationship with psychosocial variables and, compare our results with previous studies. Methods: A voluntary and self-report survey was designed, which included (AUDIT) for alcohol consumption, questions taken from the National Service for Prevention and Rehabilitation from Alcohol and Drugs' (SENDA) Survey for cannabis and cocaine use, and questions about the students' sociodemographics. Results: 559 students answered from a total of 857 (65.2\%). Risky alcohol use prevalence was $15.1 \%$, and harmful alcohol use prevalence was 5.2\%. Cannabis use prevalence was $57.2 \%$ ever in their lifetime, 38.2\% last year, and $18.5 \%$ last month. Cocaine use prevalence was $2.8 \%$ ever in their lifetime, $1.6 \%$ last year and $0.5 \%$ last month. Associations were found between substance use and our studied variables. Conclusions: Substance use among medical students from Pontificia Universidad Católica has risen considerably. However, the prevalence remains low compared to the prevalence in the rest of Chilean higher education students and similar to the reported one from studies in European and North American medical students.
\end{abstract}

Keywords: medical students; alcohol; cannabis; cocaine; substance use disorder; drug abuse.

Fecha de envío: 2021-05-28 - Fecha de aceptación: 2021-08-19

\section{Introducción}

El uso de sustancias en adolescentes y adultos jóvenes es un problema importante en Chile. Según la Encuesta Nacional de Salud
(ENS) 2016-2017, un 11,7\% de la población ha presentado un patrón de consumo riesgoso de alcohol en el último año. A su vez, el $13^{\circ}$ estudio nacional de drogas en población general

(1) Escuela de Medicina, Pontificia Universidad Católica de Chile. Santiago, Chile.

(2) Departamento de Psiquiatría, Facultad de Medicina, Pontificia Universidad Católica de Chile. Santiago, Chile.

*Autor de Correspondencia: jsantand@uc.cl 
realizado por SENDA el 2018, indica que el consumo de alcohol en jóvenes de 19 a 25 años alcanza un 53,2\% en el último mes. Por otra parte, el consumo de marihuana en la última década ha presentado un aumento significativo para el mismo rango etario, desde un 12,3\% en 2010, a 24,1\% en 2014 y a un 32,1\% en 2018 (Ministerio del Interior y Seguridad Pública, 2017).

En particular, los estudiantes de educación superior de nuestro país se han conformado como un grupo de riesgo importante para el consumo de sustancias. Esto quedó evidenciado en el "primer estudio de drogas en educación superior" realizado por SENDA. Este estudio muestra que existe una prevalencia del $68,0 \%$ de consumo de alcohol en el último mes, de los cuales un $68,1 \%$ declara haberse embriagado en el último mes. En cuanto al uso de marihuana, un $33,1 \%$ habría declarado consumo en el último mes y un 50,4\% en el último año. El consumo de cocaína alcanza una prevalencia anual de 4,9\% (SENDA, 2019).

Dentro de los estudiantes universitarios, los estudiantes de medicina son un grupo de alto riesgo (Akvardar et al., 2003). Se ha postulado que el consumo de sustancias se presenta como un amortiguador del estrés psicológico, explicado por las características de los componentes que gatillan un mecanismo de recompensa y placer (Arora et al., 2016). Según el estudio de Candido et al. (2018) las sustancias que predominan en tal población son el alcohol, el tabaco y la marihuana. En el caso particular de nuestro país, Romero et al. (2007) investigaron la prevalencia de consumo de distintas sustancias en estudiantes de medicina de la PUC, entre ellas marihuana, cocaína, anfetaminas, benzodiacepinas y antidepresivos, el año 2007. Dentro de las conclusiones, destaca una prevalencia de consumo de sustancias similar al resto de jóvenes y universitarios chilenos de aquella época, aunque menor que lo reportado por estudios en estudiantes de medicina en otros países, como Reino Unido.

El objetivo del presente estudio es determinar la prevalencia de consumo de alcohol, marihuana y cocaína en los estudiantes de medicina de la PUC. Además, se analizará la asociación entre consumo y distintas variables psicosociales, esto con el fin de poder asociar patrones de consumo y contrastar los resultados obtenidos con estudios previos en el mismo grupo, otras poblaciones de estudiantes de medicina, y la población universitaria chilena.

\section{Método}

La presente investigación corresponde a un estudio analítico, individual y transversal. El estudio se llevó a cabo durante la tercera y cuarta semana de junio de 2019, previa autorización de Dirección de Pregrado de la Escuela de Medicina UC y aprobación por parte del comité de ética institucional.
Se invitó a participar a todos los estudiantes de primero a séptimo año de medicina de la PUC ( $\mathrm{n}=857$ ) vía correo electrónico y redes sociales. Previo a la respuesta, los estudiantes aceptaron un consentimiento informado donde se explicaba la finalidad y utilidad del estudio y se aseguraba el anonimato y confidencialidad para el manejo posterior de la información. Una vez aceptado el consentimiento, los participantes procedían a responder una encuesta, que se facilitó vía Google Forms, y cuyos datos fueron exportados a Microsoft Excel en primera instancia. Respondieron un total de 559 estudiantes, lo que corresponde a un $65,2 \%$ del total. El proceso fue supervisado por el equipo investigador.

Para la detección del consumo de alcohol se utilizó el cuestionario "Alcohol Use Disorders Identification Test"(AUDIT), instrumento válido y confiable para la identificación del consumo de alcohol en población adulta chilena. Se utilizó una versión AUDIT validada para población universitaria donde se proponen puntos de corte de 6 a 8 para consumo de riesgo y 9 para consumo perjudicial (García et al., 2016).

En cuanto al consumo de marihuana y cocaína, se decidió trabajar con 3 preguntas extraídas del décimo segundo estudio nacional de drogas en población general de Chile (SENDA, 2016). En concreto se preguntó, "consumo en el último año", "consumo en el último mes" y "consumo alguna vez en la vida", de tal forma, se logró que los datos obtenidos fueran comparables con los datos poblaciones del país.

Las variables dependientes analizadas fueron consumo de alcohol, consumo de marihuana en el último mes y consumo de cocaína alguna vez en la vida. Estas se asociaron a variables independientes, recabadas en la misma encuesta. Las variables independientes fueron sexo, edad, curso, tipo de financiamiento de educación superior, vivir solo/a, reprobación de algún ramo durante la carrera, estar en tratamiento por diagnóstico previo de salud mental y ocurrencia de eventos vitales estresantes en los últimos 12 meses. Este último se definió como "fenómeno económico, social, psicológico o familiar de carácter brusco, que produce desadaptación social o distrés psicológico. Ejemplos: muerte de algún familiar o amigo cercano, separación de padres, lesión o enfermedad propia o de un cercano, término de una relación amorosa, cesantía de padre o madre, incorporación de nuevo miembro a la familia, entre otros."

Las variables categóricas se muestran como número de casos y porcentajes. El análisis estadístico de los datos obtenidos se realizó mediante el programa SPSS 17 (SPSS Inc., 2008), aplicando las pruebas de significación estadística pertinentes: diferencia de proporciones, $\mathrm{X}^{2}$, y prueba exacta de Fisher para estudiar las asociaciones entre las variables elegidas. Se consideró significativo todo valor $\mathrm{p}$ menor o igual a 0,05. 


\section{Resultados}

La encuesta fue respondida de manera voluntaria por 559 de un total de 857 estudiantes (65,2\%). Del total de encuestados, 297 fueron mujeres $(53,1 \%)$ y 262 hombres $(46,9 \%)$. El curso con mayor cantidad de respuestas fue segundo año $(n=99)$ y el con menos sexto año ( $n=62$ ). El 62,5\% de los estudiantes se encuentran en el rango etario entre 18 a 22 años, un 34,6\% entre 23 a 27 años y un 1,8\% tiene 27 años o más. Un 52,2\% tiene a su familia de origen (padre y/o madre) como fuente única de financiamiento de sus estudios. Por otro lado, el $81,7 \%$ vive con integrantes de su familia nuclear, mientras que un 9,5\% vive solo/a. El 8,1\% ha reprobado algún ramo durante su carrera académica. El 54,6\% de los encuestados declara haber vivido un evento vital estresante en los últimos 12 meses. El 24,7\% está actualmente en tratamiento por diagnóstico previo de salud mental.
De los 559 encuestados, 558 completaron el cuestionario AUDIT. Un cuestionario fue respondido de manera incompleta y se excluyó del análisis. La prevalencia de consumo de riesgo dentro de la población estudiada fue de un $15,1 \%$ ( $n=84)$, mientras del consumo perjudicial de un 5,2\% ( $n=29)$. El sexo fue la única variable que presentó asociación estadísticamente significativa con los resultados del AUDIT $(p<0,001)$. Los encuestados de sexo masculino, reportaron una prevalencia de consumo de riesgo de $19,5 \%(n=51)$ y de consumo perjudicial de $9,5 \%(n=25)$, mientras que en el género femenino se reportó prevalencias de consumo de riesgo y consumo perjudicial de $11,1 \%(n=33)$ y $1,4(n=4)$ respectivamente. No se encontraron otras asociaciones significativas entre los resultados del AUDIT y las demás variables estudiadas (Tabla 1).

Tabla 1: Prevalencia de consumo de alcohol, por 100 estudiantes, según género.

\begin{tabular}{|c|c|c|c|c|c|c|}
\hline & \multicolumn{2}{|c|}{ Femenino } & \multicolumn{2}{|c|}{ Masculino } & \multicolumn{2}{|c|}{ Total } \\
\hline & $\mathrm{n}$ & $\%$ & $\mathrm{n}$ & $\%$ & $\mathrm{n}$ & $\%$ \\
\hline Consumo de riesgo & 33 & 11,1 & 51 & 19,5 & 84 & 15,1 \\
\hline Consumo perjudicial & 4 & 1,4 & 25 & 9,5 & 29 & 5,3 \\
\hline
\end{tabular}

En cuanto al consumo de marihuana, un 57,2\% ( $n=319)$ admite haber consumido marihuana alguna vez en su vida, un $38,2 \%$ $(n=213)$ reporta haber consumido el último año, y un $18,5 \%$ $(n=103)$ el último mes. El consumo mensual reportado fue mayor en hombres, donde un $21,8 \%(n=57)$ reportó haber consumido en el último mes, comparado con un $15,5 \%(n=46)$ de las mujeres, siendo esta asociación estadísticamente significativa $(p=0,036)$. Además, quienes afirman estar en tratamiento por alguna patología de salud mental, reportan una mayor prevalencia de consumo mensual $(26,8 \%, n=37)$, comparado con quienes no reportan estar en tratamiento $(15,7 \%, n=66)$, siendo esta asociación significativa $(p=0,003)$. El resto de las variables independientes no son estadísticamente significativas.

Respecto al consumo de cocaína, la prevalencia reportada de consumo alguna vez en la vida fue de un 2,8\% $(n=16)$, en el último año un $1,6 \%(n=9)$ y en el último mes un $0,5 \%(n=3)$. La prevalencia de consumo alguna vez en la vida fue mayor en hombres, donde alcanzó un 3,4\% ( $n=9)$, comparado con el 2,4\% ( $n=7)$ en mujeres, sin ser estadísticamente significativo $(p=0,305)$. Entre quienes habían reprobado un ramo, se vieron mayores prevalencias de consumo alguna vez en la vida $(8,9 \%, n=9)$, comparados con quienes no $(2,3 \%, n=12)$, siendo esta relación estadísticamente significativa $(p=0,033)$. Haber vivido un evento vital estresante también se asoció al consumo alguna vez en la vida $(p=0,024)$, siendo el consumo más prevalente entre aquellos que habían tenido algún evento $(4,3 \%, n=13)$ comparado con quienes no $(1,2 \%, n=3)$. Finalmente, estar en tratamiento con algún diagnóstico de salud mental se asoció de manera significativa a consumo de cocaína alguna vez en la vida $(p=0,001)$, con un 9,4\% $(n=13)$ en comparación a $0,7 \%,(n=3)$. En cuanto a las otras variables independientes, no mostraron una asociación estadísticamente significativa (Tabla 2).

También se buscaron asociaciones entre las variables relacionadas al consumo de sustancias. Aquellos estudiantes con puntajes AUDIT dentro de la categoría sin riesgo, presentaron un menor consumo mensual de marihuana ( $13 \%, n=58)$, que aquellos categorizados en consumo de riesgo $(28,6 \%, n=24)$, y consumo perjudicial $(72,4 \%, n=21)$, con una asociación significativa entre ambas variables $(p<0,001)$ (Tabla 3 ).

Además, el puntaje del AUDIT se asoció significativamente al consumo de cocaína alguna vez en la vida $(p<0,001)$. Los estudiantes que presentaron consumo sin riesgo tuvieron una prevalencia menor $(1,3 \%, n=3)$ que los consumidores de riesgo $(8,3 \% n=7)$ y consumo perjudicial $(10,3 \%, n=3)$ (Tabla 4$)$. 
Calletti et al.

Tabla 2: Prevalencia de consumo de sustancias ilícitas, por 100 estudiantes, según género.

\begin{tabular}{|c|c|c|c|c|c|c|c|}
\hline \multirow[t]{2}{*}{ Sustancia } & \multicolumn{2}{|c|}{ Femenino } & \multicolumn{2}{|c|}{ Masculino } & \multirow[t]{2}{*}{$\mathbf{P}$} & \multicolumn{2}{|c|}{ Total } \\
\hline & $\mathrm{n}$ & $\%$ & $\mathrm{n}$ & $\%$ & & $\mathrm{n}$ & $\%$ \\
\hline \multicolumn{8}{|l|}{ Consumo marihuana } \\
\hline Alguna vez en la vida & 160 & 53,9 & 159 & 60,7 & 0,14 & 319 & 57,1 \\
\hline En el último año & 103 & 34,7 & 110 & 42 & 0,07 & 213 & 38,1 \\
\hline En el último mes & 46 & 15,5 & 57 & 21,8 & 0,05 & 103 & 18,4 \\
\hline \multicolumn{8}{|l|}{ Consumo cocaína } \\
\hline Alguna vez en la vida & 7 & 3,4 & 9 & 2,4 & 0,44 & 16 & 2,9 \\
\hline En el último año & 4 & 1,3 & 5 & 1,9 & 0,59 & 9 & 1,6 \\
\hline En el último mes & 1 & 0,3 & 2 & 0,8 & 0,49 & 3 & 0,5 \\
\hline
\end{tabular}

Tabla 3: Prevalencia de consumo de marihuana en el último mes, por 100 estudiantes, según consumo de alcohol.

\begin{tabular}{|c|c|c|c|c|c|}
\hline & Sí & & No & & Total \\
\hline & $\mathrm{n}$ & $\%$ & $n$ & $\%$ & $\mathrm{n}$ \\
\hline Sin riesgo & 58 & 13 & 387 & 87 & 445 \\
\hline Consumo de riesgo & 24 & 28,6 & 60 & 71,4 & 84 \\
\hline Consumo perjudicial & 21 & 72,4 & 8 & 27,6 & 29 \\
\hline
\end{tabular}

Tabla 4: Prevalencia de consumo de cocaína alguna vez en la vida, por 100 estudiantes, según consumo de alcohol.

\begin{tabular}{lccccc} 
& Sí & & No & & \\
& $\mathrm{n}$ & $\%$ & $\mathrm{n}$ & $\%$ & $\mathrm{n}$ \\
\hline Sin riesgo & 6 & 1,3 & 439 & 98,7 & 445 \\
\hline Consumo de riesgo & 7 & 8,3 & 77 & 91,7 & 84 \\
\hline Consumo perjudicial & 3 & 10,3 & 26 & 89,7 & 29
\end{tabular}

\section{Discusión}

El estudio logró una alta tasa de respuesta a las encuestas (65,2\%), lográndose una muestra representativa de los estudiantes de medicina de la PUC.

Los resultados muestran una importante prevalencia de consumo de riesgo de alcohol $(15,1 \%)$ y consumo perjudicial de alcohol $(5,2 \%)$. En comparación con el resto de los universitarios chilenos las cifras reportadas son levemente menores, con $20,1 \%$ para consumo de riesgo y $6,4 \%$ para el consumo perjudicial. En comparación con estudiantes de medicina internacionales, existen estudios que ocupan los cuestionarios AUDIT y CAGE, donde se registró en conjunto la prevalencia de consumo tanto de riesgo como perjudicial. Nuestra prevalencia conjunta fue de un $20,3 \%$, que se encuentra por encima de lo observado en estudiantes de medicina de países latinoamericanos (13,7\%, $\mathrm{n}=12809)$, y norteamericanos $(12,3 \%$, $\mathrm{n}=3975$ ), pero similar a lo reportado por estudiantes europeos
(23,8\%, $n=6036)$ (Roncero et al., 2015). El consumo de alcohol se asoció significativamente al sexo del estudiante, tal como se ha visto en numerosas publicaciones (Collel et al., 2013; Roncero et al., 2015; Merlo et al., 2017; SENDA, 2019). Por otra parte, se evidenció asociación significativa tanto entre consumo de marihuana como cocaína. No se encontraron más asociaciones entre las numerosas variables analizadas.

Respecto a la marihuana, los reportes del décimo segundo estudio de SENDA dan cuenta de un aumento sistemático del consumo de esta droga a nivel nacional, donde él y el "consumo en el último mes" es 8,7\% y el "consumo alguna vez en la vida" es de 35,7\%. Este fenómeno también se presenta en nuestro estudio. Si comparamos los resultados obtenidos con los publicados por Romero et al. (2015) realizados en la misma población, se aprecia que la prevalencia reportada de "consumo el último mes" se triplicó durante los últimos 12 años, elevándose desde un 5,1\% a un 18,1\%. El "consumo alguna vez en la vida" aumentó de un 33\% a 38,2\%. 
Al comparar la prevalencia de consumo de marihuana con estudiantes de medicina de distintas regiones del mundo, se obtuvo un resultado mayor que lo reportado en otros países latinoamericanos $(9,5 \%, \mathrm{n}=9196)$, pero similar a lo reportado en Norte América $(16,5 \%, n=5095)$ y Europa $(20,0 \%, n=2722)$ (Roncero et al., 2015).

Destaca la asociación entre el género masculino y el consumo de marihuana, descrita en estudios nacionales e internacionales (Collel et al., 2013; Roncero et al., 2015; SENDA, 2019). También destaca la asociación entre consumo y estar en tratamiento por diagnóstico de salud mental, asociación reportada en estudios internacionales (Merlo et al., 2017). Esto nos habla de un porcentaje importante de estudiantes con problemas de abuso de substancias y comorbilidades psiquiátricas, que se ha denominado en la literatura como "patología dual", dada la elevada frecuencia de esta asociación (Regier et al., 1990).

Respecto al consumo de cocaína en nuestra población se ha evidenciado un aumento en los últimos 12 años, con "consumo alguna vez en la vida" desde 1,1\% a 2,8\%, no obstante, la prevalencia es similar a la de estudiantes de medicina de países latinoamericanos $(1,88 \%, n=2038)$ y europeos $(1,0 \%, n=1937)$, aunque menor comparada con aquellos norteamericanos (3,86\%, n=1865). Al comparar con la población chilena, destaca en los resultados de SENDA una la prevalencia de "consumo alguna vez en la vida" de $5,9 \%$ y en estudios de consumo de cocaína con los estudiantes de educación superior chilenos una prevalencia de 4,9\%, siendo ambas mayores a los resultados obtenidos en nuestro estudio. Esto podría explicarse por el conocimiento médico de los efectos adversos del consumo de cocaína. Sería interesante analizar la percepción de riesgo en futuros estudios.

El consumo de cocaína se encontró relacionado a ciertas variables psicosociales. El sexo masculino nuevamente es un factor de riesgo reconocido, tanto en población universitaria (Roncero et al., 2015) y población general, como en estudios nacionales (SENDA, 2019) e internacionales (Collel et al., 2013). El haber reprobado un ramo y haber tenido un evento vital estresante también se asociaron al consumo.

Esta asociación se puede interpretar como consumo secundario a aquellos estresores (Merlo et al., 2017). Otra perspectiva, es que el consumo contribuya a los estresores y, por ejemplo, el consumo llevar a un mal rendimiento académico (Bugbee et al., 2019). Por último, estar en tratamiento por una patología de salud mental también se asoció al consumo de cocaína. Nuevamente se evidencia la existencia de este grupo de consumidores con "patología dual" (Regier et al., 1990). Probablemente estos estudiantes requieren la ayuda de equipos multidisciplinarios para el tratamiento de sus patologías, siendo este poco disponible en nuestro medio (Torales et al., 2019).
Así este estudio muestra niveles elevados de consumo tanto de alcohol, marihuana y cocaína entre los estudiantes de medicina, por lo que se hace perentorio generar iniciativas e instancias para frenar este aumento. En la PUC se han realizado esfuerzos para contrarrestar este fenómeno. El programa de autocuidado en drogas para estudiantes universitarios (PADEU) es el organismo encargado de la orientación, apoyo y tratamiento de aquellos estudiantes que requieran ayuda en este ámbito. Entre las medidas propuestas durante los últimos años destaca la nueva normativa de consumo de alcohol en la Universidad y talleres de pares educadores en salud (PES), entre otros. Se sugiere continuar generando e impulsando iniciativas y actividades de prevención, para poder detener el aumento del consumo de drogas que hemos visto en los últimos años.

Nuestro estudio presentó ciertas limitaciones en cuanto a la caracterización del consumo de sustancias ilícitas, entre ellas edad de inicio de consumo y cantidad de consumo. Sería interesante incorporar estos aspectos en futuras investigaciones.

Nos parece de alta relevancia enfatizar que el equipo de salud constituye una herramienta fundamental para la educación, prevención y tratamiento del consumo de sustancias. Es imperioso a tiempo sobre este tema ya que los estudiantes encuestados se verán enfrentados al tratamiento de pacientes con trastornos por uso de sustancias. Por lo mismo, el prevenir y tratar el consumo de sustancias en este grupo debe ser prioridad.

\section{Contribuciones y reconocimientos}

Los autores manifiestan no tener ningún conflicto de interés ni han recibido apoyo económico.

\section{Referencias}

Akvardar Y, Demiral Y, Ergor G, Ergor A, Bilici M \& Ozer OA. (2003). Substance use in a sample of Turkish medical students. Drug Alcohol Depend 72, 21-117.

Alvarado ME, Garmendia ML, Acuña G, Santis R \& Arteaga O. (2009). Validez y confiabilidad de la versión chilena del Alcohol Use Disorders Identification Test (AUDIT). Rev Med Chile 137, 11.

Arora A, Kannan S, Gowri S, Choudhary S, Sudarasanan S \& Khosla PP. (2016). Substance abuse amongst the medical graduate students in a developing country. Indian J Med Res 143, 3-101.

Bugbee BA, Beck KH, Fryer CS. \& Arria AM. (2019). Substance Use, Academic Performance, and Academic Engagement Among High School Seniors. The Journal of school health $\mathbf{8 9}, 145-156$. 
Candido FJ, Souza R, Stumpf MA, Fernandes LG, Veiga R, Santin M $\&$ Kluthcovsky A. (2018). The use of drugs and medical students: a literature review. Rev Assoc Med Bras 64, 462-468.

Colell E, Sánchez-Niubò A \& Domingo-Salvany A. (2013). Sex differences in the cumulative incidence of substance use by birth cohort. Int J Drug Policy 24, 319-25.3

De Andrade V, Pereira L, Guerra A, Nicastri S. \& Malbergier A. (2005). Comparative study of drug use among undergraduate students at the University of Sao Paulo, Sao Paulo campus in 1996 and 2001. Rev Bras Psiquiatr 27, 93-185.

Degenhardt, L, Chiu WT. Sampson N, Kessler RC. \& Anthony JC. (2007). Epidemiological patterns of extra-medical drug use in the United States: Evidence from the National Comorbidity Survey Replication, 2001-2003. Drug and Alcohol Dependence 90, 210-223.

García M, Novalbos J, Martinez J. \& O Ferrall C. (2016). Validación del test para la identificación de trastornos por uso de alcohol en población universitaria: AUDIT y AUDIT- C. Adicciones 28, 194- 204

Merlo LJ, Curran JS. \& Watson R. (2017). Gender differences in substance use and psychiatric distress among medical students: A comprehensive statewide evaluation. Subst Abus 38, 401-406.

Ministerio del Interior y Seguridad Pública (2017). Décimo segundo estudio nacional de drogas en población general de Chile 2016. Observatorio chileno de drogas. Gobierno de Chile.

Newbury-Birch D, White M, Kamali F. Factors influencing alcohol and illicit drug use amongst medical students. Drug and Alcohol Dependence 2000 59, 125-30.

Organización de Estados Americanos. (2019). Informe sobre el consumo de drogas en las Américas 2019. Comisión Interamericana para el Control del Abuso de Drogas (CICAD).

Pontificia Universidad Católica de Chile. (2015). Política de prevención del uso de drogas ilegales y del abuso de alcohol. Vida Universitaria UC. Accedido en: http://vidauniversitaria.uc.cl/documentos/ etica_uc_prevencion_uso_drogas_ilegales_abuso_alcohol.pdf el 15 de febrero de 2020.
Regier DA, Farmer ME, Rae DS, Locke BZ, Keith SJ, Judd L, Goodwin FK. (1990). Comorbidity of mental disorders with alcohol and other drug abuse. Journal of the American Medical Association 264, 2511-2518.

Romero MI, Santander J, Hitschfeld M, Labbé M \& Zamora, V. (2009). Consumo de tabaco y alcohol entre los estudiantes de medicina de la Pontificia Universidad Católica de Chile. Rev Med Chile 137, 361-368.

Roncero C, Egido A, Rodríguez L, Pérez J, Collazos F \& Casas M. (2013). Substance Use among Medical Students: A Literature Review. Actas Esp Psiquiatr 43, 21-109.

Seguel F, Santander G \& Alexandre O. (2013). Validez y confiabilidad del test de identificación de los trastornos debido al consumo de alcohol (AUDIT) en estudiantes de una universidad chilena. Cienc. enferm. 19, 1.

SENDA. (2019). Primer estudio de drogas en Educación Superior 2018, Ministerio del Interior y Seguridad Pública. Gobierno de Chile, pp. 4-18.

SPSS Inc. (2008). SPSS Statistics for Windows, Version 17.0. Chicago: SPSS Inc.

Torales J, Castaldelli-Maia JM, da Silva AG, Campos MW, González-Urbieta I, Barrios I. (2019). Even More Complex.... When Mental Disorder Meets Addiction in Youth: Dual Pathology. Curr Drug Res $\operatorname{Rev} 11,40-43$.

Trucco M, Campusano M. \& Larraín S. (1979). Un cuestionario para detectar desórdenes emocionales. Un estudio de validación preliminar. Rev Chil Neuro-Psiquiatr. 17, 5-20.

Welte JW. \& Barnes GM. (1985). Alcohol: The gateway to other drug use among secondary-school students. J Youth Adolesc. 14, 98-487. 